\title{
TWO THEOREMS ON THE MAPPING CLASS GROUP OF A SURFACE
}

\author{
JEROME POWELL
}

\begin{abstract}
The mapping class group of a closed surface of genus $>3$ is perfect. An infinite set of generators is given for the subgroup of maps that induce the identity on homology.
\end{abstract}

Let $T_{g}$ be the boundary of a 3-ball with $g$ handles, $H\left(T_{g}\right)$ be the group of all orientation preserving homeomorphisms of $T_{g}, D\left(T_{g}\right)$ be the subgroup of all homeomorphisms which are isotopic to the identity. The mapping class group $M\left(T_{g}\right)$ is the quotient group $H\left(T_{g}\right) / D\left(T_{g}\right)$. Generators for $M\left(T_{g}\right)$ are known, but a complete set of relations for $M\left(T_{g}\right)$ is only known for $g=2$ [2]. The commutator subgroup $M\left(T_{2}\right) /\left[M\left(T_{2}\right), M\left(T_{2}\right)\right]$ is $\mathbf{Z}_{10}$.

We shall prove that $M\left(T_{g}\right)$ is perfect for $g \geqslant 3$. We shall also give an infinite set of generators for the subgroup $K_{g}$ of $M\left(T_{g}\right)$ which induces the identity on homology, i.e. the kernel of the map from $M\left(T_{g}\right)$ onto $\Gamma_{g}$, Siegel's modular group [1].

THEOREM 1. The mapping class group $M\left(T_{g}\right)$, for $g \geqslant 3$, is perfect.

Proof. For $g \geqslant 3$, it is known [1] that the commutator subgroup of $M\left(T_{g}\right)$ is either $\mathbf{Z}_{2}$ or the trivial subgroup, and also that, if it were $\mathbf{Z}_{2}$, then every Dehn twist about a nonseparating curve on $T_{g}$ would be mapped onto the generator of $\mathrm{Z}_{2}$. To prove that the commutator subgroup of $M\left(T_{g}\right)$ is trivial, for $g \geqslant 3$, it is sufficient to exhibit a product of an odd number of Dehn twists about nonseparating curves that is isotopic to the identity. Such a product is given by the 15 following twists:

$$
\left(Y_{3} Y_{2} Y_{1} Z_{1}^{-1} U_{2} Z_{2}^{-1} U_{2}^{-1} Y_{2}^{-1} U_{2} Z_{1} U_{2} Z_{2} Y_{2}^{-1} U_{2}^{-1}\right) A^{-1}=\mathrm{id}
$$

where $U_{i}, Y_{i}, Z_{i}, A$ are Dehn twists about the curves $u_{i}, y_{i}, z_{i}, a$. (See Figures 1 and 2.) At the end of the paper, we will explain how to check the assertion.

Let $K_{g}$ be the kernel of the map from $M\left(T_{g}\right)$ to $\Gamma_{g}$, Siegel's modular group [3].

THEOREM 2. For $g \geqslant 3, K_{g}$ is generated by 2 types of twists:

TYPE 1. Dehn twists about separating curves (which divide the surface in such a way that one of the resulting components has genus 1 or 2).

Received by the editors June 21, 1976.

AMS (MOS) subject classifications (1970). Primary 55A05, 57E05; Secondary 57A05.

Key words and phrases. Mapping class group, identity on homology, Dehn twists. 


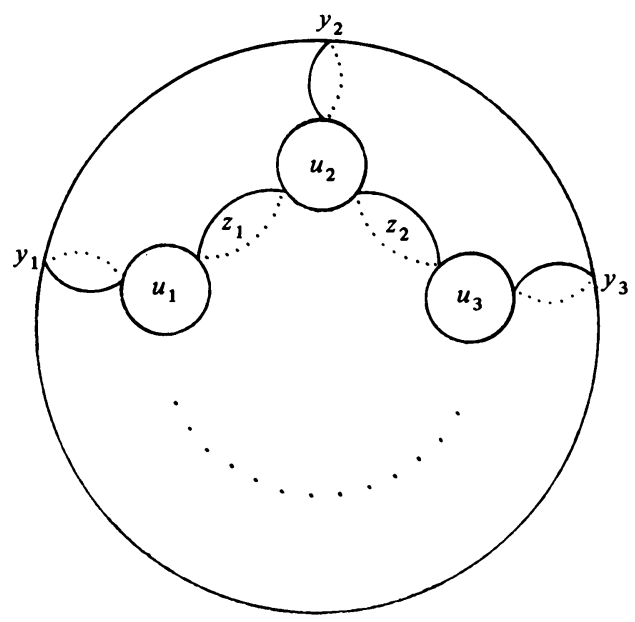

Figure 1

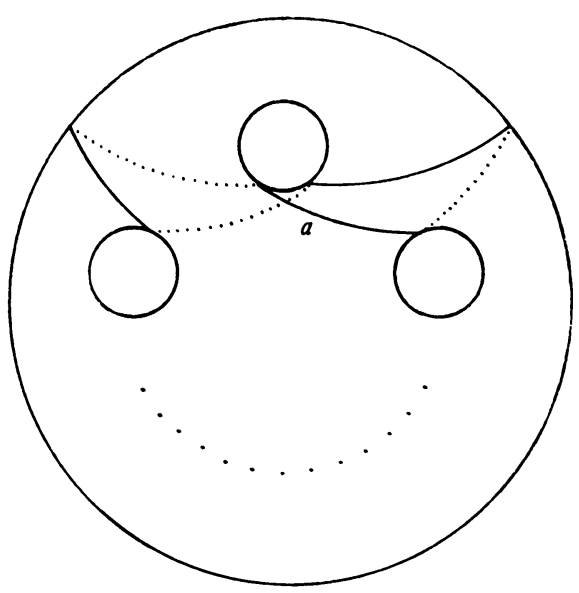

FiguRE 2

TYPE 2. "double twists", i.e. simultaneous Dehn twists $R$ and $S^{-1}$ about disjoint homologous curves $r$ and $s$ ( $r$ and $s$ divide the surface in such a way that one of the resulting components has genus 1).

Proof. Birman showed ([3, Theorem 2]) that, for $g \geqslant 3, K_{g}$ is generated by the conjugates in $M\left(T_{g}\right)$ of

$$
\begin{aligned}
& \left(Y_{1} U_{1} Y_{1}\right)^{4}, \\
& \left(Y_{1} U_{1} Z_{1} U_{2} Y_{2}\right)^{6}, \\
& \left(Y_{1} U_{1} Z_{1} U_{2} Y_{2}^{2} U_{2} Z_{1} U_{1} Y_{1}\right) Y_{1}\left(Y_{1} U_{1} Z_{1} U_{2} Y_{2}^{2} U_{2} Z_{1} U_{1} Y_{1}\right)^{-1} Y_{1}^{-1}, \\
& \left(Y_{3} Y_{2} Y_{1} Z_{1}^{-1} U_{2} Z_{2}^{-1} U_{2}^{-1} Y_{2}^{-1} U_{2} Z_{1} U_{2} Z_{2} Y_{2}^{-1} U_{2}^{-1}\right) P_{2} Z_{1}^{-1} P_{2}^{-1}, \\
& \quad \text { where } P_{2}=\left(Y_{2} U_{2} Y_{2}\right)\left(Y_{2} U_{2} Z_{2} U_{3} Y_{3}\right)^{3}\left(Y_{2} U_{2} Y_{2}\right)^{-1}
\end{aligned}
$$




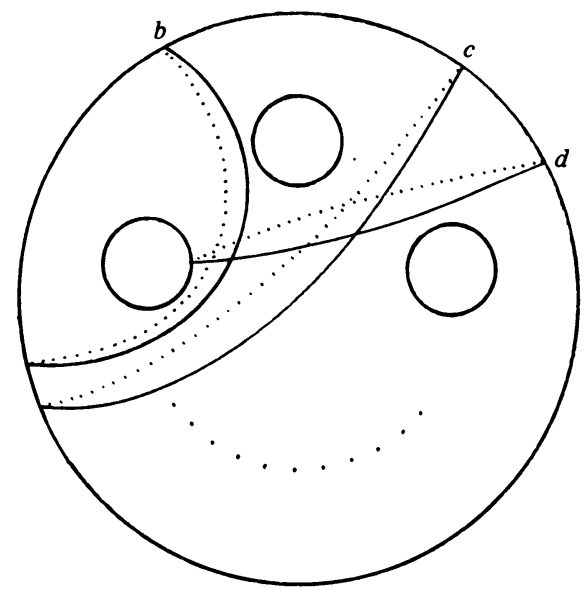

Figure 3

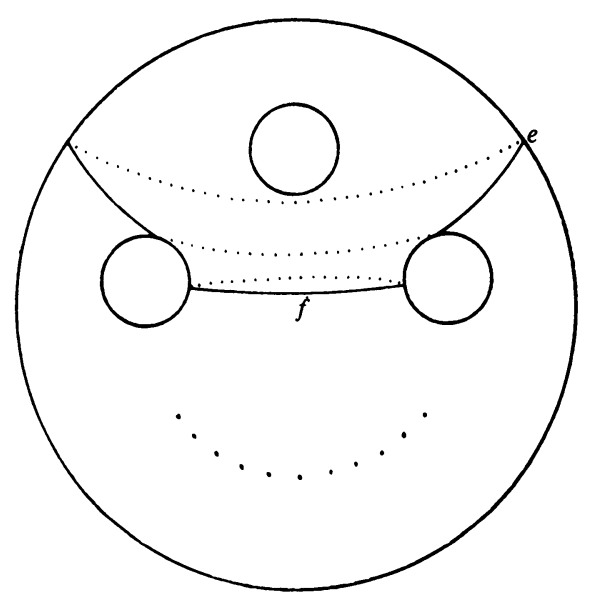

FIGURE 4

The conjugates in $M\left(T_{g}\right)$ of twists about curves are the twists about the images of the curves under the homeomorphism by which you conjugate $([4$, Lemma 1]). All twists about separating curves which divide the surface in such a way that one of the components is of genus 1 (respectively genus 2) are conjugate and all "double twists" of type 2 are conjugate. Therefore it suffices to show that the above homeomorphisms are of type 1 or type 2 .

Let $A, B, C, D, E, F$ be Dehn twists about $a, b, c, d, e, f$. We assert that the following equations hold:

$$
\begin{aligned}
& \left(Y_{1} U_{1} Y_{1}\right)^{4}=B \\
& \left(Y_{1} U_{1} Z_{1} U_{2} Y_{2}\right)^{6}=C \\
& \left(Y_{1} U_{1} Z_{1} U_{2} Y_{2}^{2} U_{2} Z_{1} U_{1} Y_{1}\right) Y_{1}\left(Y_{1} U_{1} Z_{1} U_{2} Y_{2}^{2} U_{2} Z_{1} U_{1} Y_{1}\right)^{-1} Y_{1}^{-1}=D Y_{1}^{-1} \\
& \left(Y_{3} Y_{2} Y_{1} Z_{1}^{-1} U_{2} Z_{2}^{-1} U_{2}^{-1} Y_{2}^{-1} U_{2} Z_{1} U_{2} Z_{2} Y_{2}^{-1} U_{2}^{-1}\right) P_{2} Z_{1}^{-1} P_{2}^{-1} \\
& \quad=A E^{-1}=\left(A F^{-1}\right)\left(F E^{-1}\right)
\end{aligned}
$$


The twists $B$ and $C$ are of Type 1 . The twists $D Y_{1}^{-1}, A F^{-1}, F E^{-1}$ are of Type 2.

One may verify the assertions in Theorems 1 and 2 by lengthy calculations. It suffices to show that the images of generators of $\Pi_{1}\left(T_{g}\right)$ under the sequences of twists are isotopic for each side of the equalities. This type of calculation is illustrated by Lickorish $([4$, p. 773]). Note that it is necessary to keep the base point fixed because there are nontrivial homeomorphisms of $M\left(T_{g}\right)$ such that the image of each generator of $\Pi_{1}\left(T_{g}\right)$ is freely homotopic to the generator.

\section{REFERENCES}

1. J. Birman, Abelian quotients of the mapping class group of a 2 manifold, Bull. Amer. Math. Soc. 76 (1970), 147-150; Erratum 77 (1971), 479.

2. __ Braids, links and mapping class groups, Ann. of Math. Studies, No. 82, Princeton Univ. Press, Princeton, N. J., 1974.

3. $\ldots$, On Siegel's Modular Group, Math. Ann. 191 (1971), 59-68.

4. W. B. R. Lickorish, A finite set of generators for the homeotopy group of a 2-manifold, Proc. Cambridge Philos. Soc. 60 (1964), 769-778.

Department of Mathematics, Columbia University, New York, New York 10027 